\title{
A Foods and Nutrient Comparison Study of Meals Eaten at Home vs Meals Eaten at Head Start Centers in Washington DC, USA
}

\author{
Beverly M. Copeland, PhD., RD, LD, Assistant Prof. \\ Prairie View A\&M University, USA \\ Allan A. Johnson, PhD., FASAHP, Prof. \\ Howard University, USA
}

doi: 10.19044/esj.2016.v12n33p1 URL:http://dx.doi.org/10.19044/esj.2016.v12n33p1

\begin{abstract}
The purpose of this research was to investigate the food energy and nutrient intake, and adequacy of meals served at Head Start centers versus meals consumed at home among Head Start Children. A cross-sectional descriptive survey research design was utilized. A convenience sample of 195 Head Start child and caregiver pairs was recruited from two Head Start sites within the District of Columbia (DC). Weight and height were measured and used to calculate BMI. The Centers for Disease Control and Prevention (CDC) BMI for age and gender growth charts were used to evaluate BMI levels. Dietary intakes were collected using the 24-hour food recall method, and Head Start center menus were analyzed using Nutritionist Pro Software (Axxya Systems, Redmond, WA). Data analysis was conducted using the Statistical Package for the Social Sciences (SPSS) version 19 (IBM SPSS Inc., Chicago, IL). Results of the study showed no significant difference in total calorie intakes between the overweight/obese children and normal weight children who consumed meals both at home and at Head Start centers. However, in both overweight/obese and normal weight children who consumed meals totally at home, lower nutrient intakes were noted when compared to recommended levels. It was concluded that Head Start children who consumed meals both at school and home on the day of the recall, had higher intakes of food energy and were more likely to meet recommended nutrient intakes than Head Start children who did not consume any portion of their meals at school.
\end{abstract}

Keywords: Low-income children, childhood obesity, fruit and vegetable intake, Head Start, food energy and nutrient intakes, Head Start menus, meals consumed at home vs meals eaten at Head Start 


\section{Introduction}

It has been well documented that childcare settings and their environments can influence dietary intake and health behaviors of children as more time is spent in out-of-home care (Kim et al., 2011). Dev and McBride (2013) reported on an article written by HHS (2012) which stated that more than 12 million preschool children attend childcare in America where they consume half to three-quarters of their food energy on a daily basis. Because of the long hours spent in these childcare centers, early nutritional behaviors can be formed and developed through adulthood (Dev and McBride, 2013). The National Head Start Association (NHSA) is a nonprofit organization committed to serving children and families from low-income households. Head Start was designed to help break the cycle of poverty by providing preschool children of low-income families with a comprehensive program to meet their emotional, social, health, nutritional, and psychological needs and support the families in improving their lives. These services are still being provided today (NHSA, 2016). Head Start programs are required to follow federal program performance standards, where meals are served family style, with teachers and staff sitting at the table with children and eating the same meals, except for children on special diets.

By following the standards set forth by the federal government, there is a greater chance of children being fed appropriately, this being integral to their growth and development. Funding for meals is provided by Child and Adult Care Food Program (CACFP) which is under the United States Department of Agriculture (USDA). Proper portion sizes and recommended food component must be served if reimbursement is to be claimed for meals.

Studies suggest that meals consumed in family style service, such as Head Start, are more likely to encourage healthy eating and better portion control than in other settings where children are not allowed to serve themselves (Lifsey, 2015). This study investigated the nutrient intake and adequacy of meals served in Head Start versus meals consumed at home among Head Start Children. Head Start provides a unique setting for early intervention as children can be in Head Start program up to 12 hours a day and receive three meals, depending on the program in which they participate.

\section{Methodology}

The research design utilized in this was the cross-sectional descriptive survey. One hundred and ninety-five (195) participants between the ages of two and five years and their caregivers were recruited from Head Start sites in the District of Columbia. Caregivers were referred by the Head Start Nutrition Director and teachers to the researchers. A letter explaining the project was distributed to the caregivers, who were required to sign an informed consent form. 
All data were kept confidential. No names, addresses or any other identifying information were associated with the data collected. All signed consent forms and collected data were kept in a locked file cabinet and were accessible to the investigators only.

To compare the food energy and nutrient intakes of children who consumed their meals at Head Start compared to those who consumed their meals at home, the following data were collected - weight and height, eating patterns and dietary intakes, 24-hour food recalls, and center menus.

Weights and heights of the children were measured using standard anthropometric procedures as described by Lohman et al. (1988). Body mass index (BMI) was calculated using the following formula: weight $(\mathrm{kg}) /$ height $^{2}$ (m). Weight/height status of the children was assessed using the 2000 CDC age and gender specific BMI for age charts (http://www.cdc.gov/growthcharts/cdc_charts.htm). Children were classified as underweight if their BMI levels were below the $5^{\text {th }}$ percentile, normal weight if their BMI levels were between the $5^{\text {th }}$ and the $85^{\text {th }}$ percentiles, and overweight/obese if their BMI levels were at or above the $85^{\text {th }}$ percentile. The eating practices and dietary intakes of the children were collected from the caregivers using the 24-hour food recall following the USDA Automated Multiple Pass Method (http://handle.nal.usda.gov/10113/23049). Head Start center menus were collected from center personnel. Nutritionist Pro software (Axxya Systems, Richmond, WA) was used to calculate food energy and nutrient intakes of the children, and the food energy and nutrient content of the menus.

\section{Data Analysis}

The data collected were analyzed using the Statistical Package for the Social Sciences (SPSS), version 19 (IBM SPSS Inc., Chicago, Illinois). Chisquare tests were used to compare meals eaten at home versus meals eaten at Head Start centers

\section{Results}

Results indicated that of the 195 children, 2.1\% (4) were underweight, $62.6 \%$ (122) were of normal weight, and $35.4 \%$ (69) were overweight or obese. Table 1 shows the child demographics by their BMI status. The prevalence of overweight/obesity was higher in girls (52.2\%). The majority of the overweight/obese children (62.3\%) were between the ages of 36 and 59 months. 
Table 1. Child Demographics by BMI Status

Underweight $^{1} \quad$ Normal Weight $^{2} \quad$ Overweight/Obese $^{3} \quad$ Total

Number (\%) Number (\%) Number (\%)
Number (\%)

\begin{tabular}{|c|c|c|c|c|}
\hline \multicolumn{5}{|l|}{ Gender } \\
\hline Boy & $2(50.0)$ & 67 (54.9) & 33 (47.8) & 102 (52.3) \\
\hline Girl & $2(50.0)$ & $55(45.1)$ & $36(52.2)$ & 93 (47.7) \\
\hline Total & $4(100.0)$ & $122(100.0)$ & $69(100.0)$ & $195(100.0)$ \\
\hline Age & & & & \\
\hline 24-35 Months & $2(50.0)$ & 32 (26.2) & 15 (21.7) & 49 (25.1) \\
\hline 36-47 Months & $1(25.0)$ & $44(36.1)$ & $25(36.2)$ & 70 (35.9) \\
\hline 48-59 Months & $0 \quad(0.0)$ & $32(26.2)$ & $18(26.1)$ & $50(25.6)$ \\
\hline $\begin{array}{l}60 \text { months or } \\
\text { more }\end{array}$ & $1(25.0)$ & 14 (11.5) & 11 (15.9) & 26 (13.3) \\
\hline Total & $4(100.0)$ & $122(100.0)$ & $69(100.0)$ & $195(100.0)$ \\
\hline
\end{tabular}

Table 2 shows the dietary intakes of children who consumed meals at school and at home on the day of recall, by BMI status. With the exception of total fat, protein, and fruits, intakes were higher in the overweight/obese children. Intakes of vitamin A and calcium were significantly higher in the overweight/obese children. No other significant differences were found.

Table 2. Dietary Intakes of Children who Consumed Meals at Both Home and School by BMI Status

\begin{tabular}{cccc} 
Dietary Intake & $\begin{array}{c}\text { Normal } \\
\text { Weight }\end{array}$ & Overweight/Obese $^{2}$ & Significance Level* $^{*}$ \\
Mean \pm SEM & Mean \pm SEM & \\
Food Energy (kcal) & $1565 \pm 28.8$ & $1588 \pm 45$ & 0.326 \\
Total Fat (g) & $47 \pm 1.5$ & $46 \pm 2.0$ & 0.36 \\
Satd. Fat (g) & $18 \pm 0.72$ & $20 \pm 1.1$ & 0.118 \\
Protein (g) & $67 \pm 1.6$ & $66 \pm 2.2$ & 0.385 \\
Vitamin A (IU) & $4919 \pm 406.5$ & $7101 \pm 905.5$ & 0.015 \\
Vitamin C (mg) & $96 \pm 7.2$ & $100 \pm 10.6$ & 0.369 \\
Iron (mg) & $11 \pm 0.41$ & $12 \pm 0.78$ & 0.249 \\
Calcium (mg) & $1075 \pm 32.7$ & $1235 \pm 48.4$ & 0.003 \\
Fiber (g) & $14 \pm 0.49$ & $14.7 \pm 0.65$ & 0.421 \\
Sodium(mg) & $2100 \pm 75.6$ & $2202 \pm 110.9$ & 0.217 \\
Sugar (g) & $110 \pm 2.80$ & $\pm 118 \pm 4.30$ & 0.07 \\
Vegetables (servings) & $1 \pm 0.24$ & $2 \pm 0.29$ & 0.268 \\
Fruits (servings) & $3.6 \pm 0.22$ & $3.5 \pm 0.31$ & 0.426 \\
\hline${ }^{1}$ BMI for age and gender between the $5^{\text {th }}$ and the $85^{\text {th }}$ percentiles on the 2000 CDC BMI for age charts \\
${ }^{2}$ BMI for age and gender at or above the $85^{\text {th }}$ percentile on the 2000 CDC BMI for age charts \\
*Significance levels below 0.05 indicate significant differences in the means of the two groups
\end{tabular}

Table 3 shows the dietary intakes of children who consumed their meals at home on the day of the recall by their BMI status. Children who were overweight/obese tended to have lower dietary intakes except for vitamins A and $\mathrm{C}$, iron, fiber, sugar, and fruits. Intakes of vitamin C, fiber, and fruits were 
significantly higher in overweight/obese children, while that of saturated fat was significantly lower.

Table 3. Dietary Intakes from Meals Consumed Totally at Home by BMI Status

Dietary Intakes $\quad$ Normal Weight ${ }^{1} \quad$ Overweight/Obese $^{2} \quad$ Significance

\begin{tabular}{cccc} 
& & Level* \\
\hline Food Energy (Kcal) & $1380 \pm 80.8$ & Mean \pm SEM & \\
Total Fat (g) & $48 \pm 3.82$ & $1353 \pm 134.4$ & 0.429 \\
Satd. Fat (g) & $19 \pm 1.68$ & $43 \pm 6.6$ & 0.216 \\
Protein (gm) & $60 \pm 4.0$ & $13 \pm 2.0$ & 0.0235 \\
Vitamin A (IU) & $3781 \pm 768.3$ & $5315 \pm 1409.7$ & 0.428 \\
Vitamin C (mg) & $66 \pm 10.22$ & $113 \pm 24.4$ & 0.151 \\
Iron (mg) & $11 \pm 1.03$ & $13 \pm 1.70$ & 0.0215 \\
Calcium (mg) & $707 \pm 54.4$ & $588 \pm 52.5$ & 0.27 \\
Fiber (g) & $10 \pm 0.8$ & $14 \pm 2.0$ & 0.0625 \\
Sodium (mg) & $2061 \pm 221.5$ & $1928 \pm 245.8$ & 0.009 \\
Sugar (g) & $88 \pm 6.9$ & $89 \pm 10.0$ & 0.353 \\
Vegetables (servings) & $1 \pm 0.2$ & $1 \pm 0.3$ & 0.457 \\
Fruits (servings) & $2 \pm 0.3$ & $3 \pm 0.59$ & 0.287 \\
${ }^{1}$ BMI for age and gender between the $5^{\text {th }}$ and the $85^{\text {th }}$ percentiles on the 2000 CDC BMI for age charts \\
${ }^{2}$ BMI for age and gender at or above the $85^{\text {th }}$ percentile on the 2000 CDC BMI for age charts \\
*Significance levels below 0.05 indicate significant differences in the means of the two groups
\end{tabular}

In Table 4, the dietary intakes of children whose total food intakes included meals eaten at home and at school are compared with those of children whose food intakes included meals eaten at home only on the day of the food recall. In children with normal weights who consumed their meals at school and at home, intakes of food energy, vitamin C, calcium, fiber, sugar, and fruits were significantly higher than those whose food intakes came only from foods consumed at home. Overweight/obese children who consumed foods both at school and at home on the day of the recall had significantly higher intakes of food energy, saturated fat, calcium, and sugar than children who consumed foods at home only.

Table 4. Dietary Intakes from Foods Consumed at School and Home versus at Home only Stratified by BMI Status

\begin{tabular}{cccc}
$\begin{array}{c}\text { Intakes of Normal } \\
\text { Weight Children }\end{array}$ & $\begin{array}{c}\text { Foods Consumed at } \\
\text { School and at Home } \\
\text { Mean } \pm \text { SEM }\end{array}$ & $\begin{array}{c}\text { Foods Consumed at } \\
\text { Home Only } \\
\text { Mean } \pm \text { SEM }\end{array}$ & $\begin{array}{c}\text { Significance } \\
\text { Level* }\end{array}$ \\
\hline Food Energy (Kcal) & $1565 \pm 29.8$ & $1380 \pm 80.8$ & 0.019 \\
Total Fat (g) & $47 \pm 1.5$ & $48 \pm 3.8$ & 0.350 \\
Satd. Fat (g) & $18 \pm 0.7$ & $19 \pm 1.7$ & 0.347 \\
Protein (g) & $67 \pm 1.6$ & $60 \pm 4.0$ & 0.057 \\
Vitamin A (IU) & $4919 \pm 406.4$ & $3780 \pm 768.3$ & 0.086 \\
Vitamin C (mg) & $96 \pm 7.3$ & $66 \pm 10.2$ & 0.0185 \\
Iron (mg) & $11 \pm 0.4$ & $11 \pm 1.0$ & 0.320 \\
Calcium (mg) & $1075 \pm 32.7$ & $707 \pm 54.3$ & 0.000 \\
Fiber (g) & $14 \pm 0.5$ & $10 \pm 84$ & 0.000 \\
Sodium (mg) & $2100 \pm 76.6$ & $2061 \pm 221.5$ & 0.434
\end{tabular}




\begin{tabular}{cccc} 
Sugar (g) & $111 \pm 2.8$ & $88 \pm 6.9$ & 0.002 \\
Fruit (servings) & $4 \pm 0.2$ & $2 \pm 0.3$ & 0.000 \\
Vegetables (servings) & $1.4 \pm 0.2$ & $1 \pm 0.2$ & 0.232 \\
& Intakes of Overweight/Obese Children & \\
\hline Food Energy(Kcal) & $1588 \pm 45.4$ & $1353 \pm 134.4$ & 0.017 \\
Total Fat (g) & $46 \pm 2.0$ & $43 \pm 6.6$ & 0.328 \\
Satd. Fat (g) & $20 \pm 1.1$ & $13 \pm 2.0$ & 0.003 \\
Protein (g) & $66 \pm 2.2$ & $58 \pm 5.3$ & 0.105 \\
vitamin A (IU) & $7102 \pm 905.5$ & $5315 \pm 1409.8$ & 0.159 \\
Vitamin C (mg) & $100 \pm 10.6$ & $113 \pm 24.4$ & 0.279 \\
Iron (mg) & $12 \pm 0.8$ & $13 \pm 1.7$ & 0.265 \\
Calcium (mg) & $1235 \pm 48.4$ & $588 \pm 52.5$ & 0.000 \\
Fiber (g) & $14 \pm 0.65$ & $15 \pm 2.0$ & 0.428 \\
Sodium (mg) & $2203 \pm 110.8$ & $1928 \pm 245.8$ & 0.127 \\
Sugar (g) & $118 \pm 4.2$ & $89 \pm 9.9$ & 0.0015 \\
Vegetable (servings) & $2 \pm 0.3$ & $1 \pm 0.3$ & 0.247 \\
Fruit (servings) & $4 \pm 0.3$ & $3 \pm 0.6$ & 0.297
\end{tabular}

\section{Discussion}

It has long been reported and agreed upon that adequate consumption of fruits and vegetables is protective against overweight/obesity, and provides dietary fiber that is linked to lower incidence of cardiovascular disease and better weight control in adulthood (Slavin and Lloyd, 2012). This finding is in line with caregivers' self-reported servings of fruits and vegetables to children in this study. Ninety percent of the caregivers reported serving fruit and vegetables daily. However, no significant relationship was found between the number of daily servings of fruit and vegetables and child BMI status.

The Dietary Guidelines for Americans 2015-2020 recommends food energy intakes of 1000-1400 kilocalories for children between 2 and 3 years of age, and 1200-1600 kilocalories for children between 4 and 5 years of age. Included in the Dietary Guidelines, also, are recommendations of 14 to 19 grams of fiber per 1000 kilocalories for children aged 2 to 3 years, and 19 to 23 grams of fiber per 1000 kilocalories for those aged 4 to 8 years. Dietary fiber has been well documented to be protective against obesity. Kendall et al. (2010), and Slavin \& Lloyd (2012) reported that studies have linked higher intakes of dietary fiber to improved management of body weight and lowering of cholesterol and that dietary fiber intake in childhood may be useful in preventing and treating obesity. The average fiber intake in this study was 13 grams and was found to be lower than the recommended intakes of 14 grams for every 1000 calories in both the normal-weight and overweight/obese children in the current study. To meet the recommendations, an average of 18 to 21 grams of dietary fiber intake was required.

It has been documented that fruits and vegetables are essential in providing vitamins and minerals for growth and development (Miller et al., 
2011). The Dietary Guidelines for Americans 2015-2020 recommend 1 $1 \frac{1}{2}$ cups of fruits and vegetables for 1 to 3-year-olds and 2 cups of fruits and 21/2 cup vegetables for children between the ages of 4 and 8 years. The results of this study showed children in the normal weight group who had food intake data for both school and home, had moderately low intakes of vegetables $(1 \pm 0.2$ servings/day). Children within the overweight group had slightly higher intakes of vegetables ( $2 \pm 0.3$ servings/day). However, servings in both groups were below the 2015-2020 dietary guidelines of 11/2 cups for 1 to 3-year-olds and $2 \frac{1}{2}$ cups for 4 to 8 -year-olds. This was slightly higher than the average intake reported by the USDA dietary guidelines, which reported an average intake of less than 1 serving between the ages of 3 and 5 years for children within the US (https://health.gov/dietaryguidelines/2015/resources/20152020_Dietary_Guidelines.pdf).

Miller et al. (2011) reporting on a study conducted by Casey et al. (2001) indicated that children from low-income, food-insufficient households, and minority children are at increased risk of inadequate fruit and vegetable intakes. This, they said, may be attributable to limited access to fruits and vegetables. However, limited access to fresh fruits and vegetables was not found to be a contributing factor to the inadequate consumption in this sample, as the overwhelming majority of the caregivers reported having access to fresh fruits and vegetable markets within their communities. Fruit intakes among both groups were within recommended intakes but were mostly provided by fruit juice consumption.

Among children who consumed all of their meals at home, the average food energy intake among the normal weight children was $1380 \pm 81 \mathrm{kcal}$ and $1353 \pm 134 \mathrm{kcal}$ among the overweight/obese children. There were significant differences in the consumption of saturated fat, vitamin C, fiber and fruits between overweight/obese and normal weight children. Children within the overweight/obese group, who consumed all of their meals at home, consumed less saturated fat, and higher intake of vitamin C, fiber and fruits than children within the normal weight group. This finding deviates from a study conducted by LaRowe et al. (2010) who found no significant difference between the intakes of Head Start children who consumed a portion of their meals at Head Start and those who consumed their meals from home. It is worth noting that saturated fat intake in both groups was higher than the recommended 10 percent of calories with $43 \%$ from the overweight group and $38 \%$ from the normal weight group.

It is also worth noting that intakes of vegetables, fiber and calcium did not meet recommendations in either group, and sodium intake was higher than what is recommended (1400-1900 mg) in both groups. The Dietary Guidelines for Americans 2015-2020 recommends limiting the amount of added sugar considered acceptable for a healthy diet. It is recommended that preschoolers 
with daily caloric intakes of 1,200 to 1,400 calories consume no more than 120 to 140 calories as added sugar a day; and children aged 4-8 with a daily caloric intake of 1,600 calories consume no more than 160 calories, indicating that 10 percent of food energy intakes should come from added sugar. Sugar intakes in both the normal weight $118 \pm 4.2$ grams (27 to 29 tsp) and overweight/obese $89 \pm 9.9$ grams (19 to 24 tsp) groups within this study were within the recommended levels within this study.

There were significant differences found between intakes from meals consumed both at school and home and meals consumed at home only. Meals eaten at school combined with meals consumed at home provided an average of 200 calories more than meals that were only consumed at home in both the normal weight and the overweight/obese groups. Likewise within the normal weight group, intakes of vitamin $\mathrm{C}$, calcium, fiber, fruits and sugar were significantly higher in children who consumed food both at school and home than in the group of children who consumed all meals from home. Further, participants did not meet the recommended calcium intake (500 $\mathrm{mg}$ for children aged 2 to 3 years, and $800 \mathrm{mg}$ for those aged 4 to 8 years) for most children who consumed all meals from home within either group. Within the overweight/obese group, intakes of food energy and most micro- and macronutrients (fat, protein, vitamin A, vitamin $\mathrm{C}$, and calcium) were higher in the children who consumed meals both at school and home than those who consumed meals at home only. Overall, Head Start children who consumed meals at school had higher intakes of food energy and were more likely to meet recommended nutrient intakes than Head Start children who did not consume any of their meals at school. Also, children who consumed meals both at school and at home were more likely to meet the recommended intakes of food energy, calcium, and micronutrients than children who consumed all portions of their meals at home on the day of the recall. This finding deviates from those of LaRowe et al. (2010) who reported no difference in the mean energy intakes of children whose dietary intakes occurred at Head Start Program sites versus those whose dietary intakes occurred at home. In the current study, no significant differences were found between overweight/obese and normal-weight children's energy intakes.

Overall, the results indicate that Head Start continues to play an important role in nutritional intake of the population it serves. With this study showing that children were more likely to meet recommended nutrient intakes if at least a portion of their daily meals were eaten at the Head Start center, continued funding and support of Head Start programs is integral to meeting the needs of the underserved and minority groups that Head Start serves. 


\section{Conclusion}

In this study of the comparison of meals eaten at home vs meals are eaten at Head Start Centers, children who consumed all portions of their meals at home did not meet the recommended intakes of vegetables, fiber, and vitamin C. The fruit intake recommendation was met but was mostly consumed from fruit juices. Children who consumed meals both at home and at the Head Start center had higher intakes of fruits and vegetables and were more likely to meet the 1 cup recommended intake. The results also showed that Head Start children who consumed meals both at school and at home on the day of the recall, had higher intakes of food energy, calcium, vitamin $\mathrm{C}$, and fiber and were more likely to meet recommended nutrient intakes than those who did not consume any portion of their meals at school.

\section{References:}

1. Administration for Children and Families. Pathways and partnerships for child-care excellence. http://www.acf.hhs.gov/programs/ccb/ta/pubs/pathways/pathways_part nerships_v1.pdf. Updated 2010. Accessed October 6, 2012.

2. Axxya Systems LLC, 2491 152nd Ave NE, Redmond, WA 98052. http://nutritionistpro.com/

3. IBM SPSS INC, Chicago Illinois, version 19

4. Bliss, (2004) Researchers produce innovation in dietary recall, USDA, http://handle.nal.usda.gov/10113/23049

5. Casey et al. (2001). Children in food-insufficient, low-income families: prevalence, health, and nutrition status. Archives Pediatric Adolescence Medicine, 155, 508-514

6. Centers for Disease Control and Prevention, (2010) Growth Charts. http://www.cdc.gov/growthcharts/cdc_charts.htm

7. Dev, D. A., and McBride, B.A., Academy of Nutrition and Dietetics Benchmarks for Nutrition in Child Care 2011: Are Child-Care Providers across Contexts Meeting Recommendations? Journal of the Academy of Nutrition and dietetics, Vol 113, 1346-1353

8. Kendall et al. (2010). The link between dietary fiber and human health. Food Hydrocolloids, 24, 42-48.

9. Kim, et al. ( 2012). Is There a Difference Between Center and Home Care Providers' Training, Perceptions, and Practices Related to Obesity Prevention?

10. Maternal Child Health Journal (2012) 16: 1559.

11. LaRowe et al. (2010). Dietary intakes and physical activity among preschool-aged children living in rural American Indian communities prior to a family-based healthy lifestyle intervention. Journal of the American Dietetic Association, 7, 1049-1057. 
12. Lifsey, S., (2015). Pass the Peas, Please: The Benefits of FamilyStyle Meals. Health Policy blog. "Family-Style meals" Office of Head Start National Centres, Health services newsletter, 2015 3(3)

13. Lohman et al. (1988). Anthropometric standardization Reference Manual. Champaign, Illinois: Human Kinetics Publishers.

14. Miller et al. (2011). Children's daily fruit and vegetable intake: associations with maternal intake and child weight status. Journal of Nutrition Education and Behavior, 43, 396-400.

15. National Head Start Associations, 2016, https://www.nhsa.org/aboutus/mission-vision-history

16. USDA Dietary Guidelines For Americans 2015-2020, 8th edition https://health.gov/dietaryguidelines/2015/resources/20152020_Dietary_Guidelines.pdf

17. Slavin, JL., and Lloyd, B., (2012). Health Benefits of Fruits and Vegetables. Advances in Nutrition, Vol 3: 506-516 